\title{
Magneto-Hydrodynamic Flow of a Viscous Fluid in a Channel with a Porous Bounding Wall of Different Permeabilities
}

\author{
Nidhi Pandya \\ Department of Mathematics \& Astronomy \\ Lucknow University, Lucknow, India \\ E-mail: dr.nidhi23@gmail.com \\ Pravin Kumar Srivastava \\ Department of Applied Science \& Humanities \\ Bundelkhand Institute of Engineering \& Technology, Jhansi, India \\ Corresponding author: drpravinsrivastava@yahoo.co.in \\ Ramesh Yadav \\ Department of Mathematics \\ Babu Banarasi Das National Institute of Technology and Management, Lucknow, India \\ E-mail: ramesh.yadav.maths@gmail.com \\ R. P. Pandey \\ Department of Applied Science \& Humanities \\ Bundelkhand Institute of Engineering \& Technology, Jhansi, India \\ E-mail: pandeyravi52@yahoo.com
}

(Received October 22, 2018; Accepted April 3, 2019)

\begin{abstract}
In this paper, the effects of permeability parameter on the magneto-hydrodynamic laminar flow of a viscous incompressible fluid in a channel with a porous bounding wall have been investigated. A transverse magnetic field has been applied in the channel flow of viscous fluid and the stream function is used to reduce the governing partial differential equations into non-linear ordinary differential equation which is solved by applying Perturbation method. Numerical simulation is used to analyze the problem and the graphs have been plotted using MATLAB. It has been observed that an increase of magnetic parameter increases the velocity of the viscous fluid while the velocity decreases with the increase of permeability parameter. The flow of viscous fluid has also been investigated with the variation of Reynolds number and the slip coefficient.
\end{abstract}

Keywords- Porous medium, MHD Flow, Reynolds number, Slip coefficient, Laminar flow.

\author{
Nomenclature \\ $h$ Height of the channel \\ $x$ Horizontal distance from the channel entrance \\ $y$ The distance along coordinate axis which is perpendicular to the channel walls measured from the non- \\ porous wall \\ $u$ Velocity component in the $x$-direction \\ $U$ Average velocity of fluid over the channel at entrance of the channel \\ Re Wall Reynolds number \\ $v$ Velocity component in the $y$-direction \\ $K$ Permeability parameter
}


International Journal of Mathematical, Engineering and Management Sciences

Vol. 4, No. 3, 708-728, 2019

https://dx.doi.org/10.33889/IJMEMS.2019.4.3-056

$B$ Magnetic field

$M$ Hartmann number (also known as magnetic parameter)

$H_{0}$ Strength of the magnetic field

\section{Greek Symbols}

$\rho$ Density of the viscous fluid

$\eta$ Non-dimensional distance in the $y$-direction, $y / h$

$v$ Kinematic coefficient of viscosity

$\sigma$ Electrical conductivity

$\Psi$ Stream function

$\phi \quad$ Slip coefficient, $\sqrt{ } k / \alpha h$

$\Delta p$ Non-dimensional pressure drop, $2[p(0, \eta)-p(x, \eta)] /\left(\rho u_{0}^{2}\right)$

\section{Introduction}

The study of a steady laminar flow of electrically conducting viscous fluids confined in a channel with the porous walls has been subject of intensive study because of its applications in industrial and geophysical situations such as the prevention of the boundary layer separation with injection or suction, magneto hydrodynamics generators, membrane separation, the filtration process, electrochemical engineering and other biological transport systems such as plasma studies, blood flow problems etc. Many researchers have solved the problem of laminar flow of incompressible viscous fluids over and through porous walls of different permeabilities under steady-state situations. Berman (1953) has analyzed the laminar flow of a viscous fluid in a channel with the porous bounding walls and solved the Navier-Stokes equations with suitable boundary conditions. He has discussed a complete description of the flow of viscous fluid flowing in a channel with equally porous walls having a rectangular cross-section and found that the velocity components and the pressure depend on position coordinates, channel dimensions, and fluid properties. In the past analysis, several researchers used boundary conditions with no slip. But the experimental results reveal that there exists a slip velocity at the bounded porous surface which is connected with the occurrence of a thin layer of stream wise fluid that is moving just below the porous surface. The results obtained in our problem reduce to the results of Berman when the Hartmann number is zero. Terrill (1965) has discussed the problem of laminar flow in a uniformly porous channel with large injection and used the method of inner and outer expansions which includes the viscous layer. Terrill and Shrestha (1966) have investigated a laminar flow through a channel with uniformly porous walls of different permeabilities. They have obtained a perturbation solution for the case of suction at one wall and injection at the other wall by choosing the velocity differences of two walls as a perturbation parameter. They confirmed the asymptotic and perturbation solutions with the exact solutions.

Vafai and Kimi (1990) have investigated fluid mechanics of an interface region between a porous medium and a fluid layer-an exact solution. Makinde and Osalusi (2006) have discussed the variation of a magnetic field on the flow of viscous incompressible fluid flowing in a channel with permeable boundaries having a slip in the steady state. They used Perturbation method for the solution of non-linear differential equations and found that the velocity of the fluid decreases due to an increase in the magnetic field as well as with the slip on the permeable boundaries. They have also found that the wall slip affects the flow inversely near the channel walls. Ganesha and Krishnambal (2006) have investigated magneto-hydrodynamic flow in the channel with two parallel plates, of a viscous fluid in the presence of a transverse magnetic field when the fluid is 
International Journal of Mathematical, Engineering and Management Sciences

Vol. 4, No. 3, 708-728, 2019

https://dx.doi.org/10.33889/IJMEMS.2019.4.3-056

moving at the same rate through both the walls of a channel. Abdou et al. (2007) have considered the laminar flow of micropolar fluids about a cone in the presence of a magnetic field with mixed thermal boundary conditions. Muthuraj and Srinivas (2009) have discussed the effects of a magnetic field and the slip on the wall on the steady flow of fluid flowing in the channel between a parallel flat wall and a long wavy wall with Soret effect. They found that the effect of increasing suction parameter suppresses the velocity while it enhances the fluid temperature.

The flow of fluid in a porous region occur in a wide range of engineering and geophysical applications such as in the flow of oil through porous rocks, drug permeation through human skin, the spreading of chemicals and fertilizers in the soil and in the industries like material and chemical, the fluid saturating the porous regions is non-Newtonian. Recently Abou-Zeid (2009) has studied the numerical treatment of the heat and the mass transfer of MHD flow of Carreau fluid with diffusion and chemical reaction through a non-Darcy porous medium. He has obtained the solution of partial differential equations by finite difference technique. Prasad and Kumar (2011) have studied the flow of a hydro magnetic fluid through the porous medium between permeable beds under an exponentially decaying pressure gradient. Mahanta (2012) has analyzed numerical study on the heat transfer of a non-Newtonian fluid flow over stretching surface with variable viscosity in a uniform magnetic field. Hosseini et al. (2013) have studied the nonNewtonian fluid flow in an axi-symmetric channel with porous walls. Jafaryar et al. (2014) have studied analytically the laminar flow through expanding or contracting gaps with porous walls. Akeem et al. (2014) have investigated the heat and mass transfers on MHD oscillatory slip flow in a channel with non-uniform wall temperature that is filled with porous medium. The exact solutions of the velocity, the temperature and the concentration have been obtained. Naikoti and Vadithya (2014) have studied thermal radiation effects on a magneto hydrodynamic flow and heat transfer in a channel with porous walls of different permeabilities. Zhang et al. (2014) obtained perturbation asymptotic solutions for asymmetric laminar flow in porous channel with expanding and contracting walls and shown that Reynolds number plays an important role in the flow behavior. Yadav and Joseph (2017) have analyzed the numerical investigation of the heat transfer along the symmetrical porous wedge and discussed the effects of radiation in the presence of variable fluid viscosity of flow.

Bujurke et al. (2010) have analyzed the laminar flow in a channel with porous bounding walls. They have investigated the effect of the magnetic field and the permeability parameter on the fluid flowing in a channel surrounded by the porous walls. The effects of various parameters like slip coefficient, Reynolds number, etc., have been shown graphically. Reddy et al. (2017) have discussed the MHD flow of a Newtonian fluid through a porous medium in the planer channel. They have used perturbation method for solving governing equations and analyzed the effects of various physical parameters graphically. Ramakrishnan and Sathish (2018) discussed the effects of the porous parameter on MHD flow between porous walls of finite thickness with different permeabilities and shown that the flow of the viscous fluid between porous walls depends upon the wall thickness. Ramadevi et al. (2018) have used the regular perturbation technique for the unsteady motion of Casson fluid over a wavy surface under the effect of magnetic field. The problem of an unsteady mixed convective flow of micro-polar fluid in the presence of transverse magnetic field has been discussed by Dey (2019). He has solved the governing equations of motion analytically using the perturbation method. 
International Journal of Mathematical, Engineering and Management Sciences

Vol. 4, No. 3, 708-728, 2019

https://dx.doi.org/10.33889/IJMEMS.2019.4.3-056

\section{Mathematical Formulation}

Let us consider the steady incompressible laminar flow of a viscous fluid in a channel of width $h$, which is bounded by one porous wall and a solid surface under the influence of a transverse magnetic field. The induced magnetic field has been neglected in the flow since the magnetic Reynolds number is so small. Let $u$ and $v$ be the components of velocity in $x$-direction and $y$ direction respectively. Then the governing equations of the continuity and linear momentum in the presence of magnetic field for this problem are

$$
\begin{aligned}
& \frac{\partial u}{\partial x}+\frac{\partial v}{\partial y}=0 \\
& u \frac{\partial u}{\partial x}+v \frac{\partial u}{\partial y}=-\frac{1}{\rho} \frac{\partial p}{\partial x}+\frac{\mu}{\rho}\left(\frac{\partial^{2} u}{\partial x^{2}}+\frac{\partial^{2} u}{\partial y^{2}}\right)-\frac{\sigma B^{2}}{\rho} u-\frac{\mu}{K} u \\
& u \frac{\partial v}{\partial x}+v \frac{\partial v}{\partial y}=-\frac{1}{\rho} \frac{\partial p}{\partial y}+\frac{\mu}{\rho}\left(\frac{\partial^{2} v}{\partial x^{2}}+\frac{\partial^{2} v}{\partial y^{2}}\right)-\frac{\sigma B^{2}}{\rho} v-\frac{\mu}{K} v
\end{aligned}
$$

where $\sigma$ is the electrical conductivity and $B\left(=\mu_{e} H_{0}\right)$ is the electromagnetic induction, $\mu_{e}$ being the magnetic permeability and $H_{0}$ is the intensity of the magnetic field.

Taking $\eta=y / h$, equations (1)-(3) reduce to

$$
\begin{aligned}
& \frac{\partial u}{\partial x}+\frac{1}{h} \frac{\partial v}{\partial \eta}=0 \\
& u \frac{\partial u}{\partial x}+\frac{v}{h} \frac{\partial u}{\partial \eta}=-\frac{1}{\rho} \frac{\partial p}{\partial x}+v\left(\frac{\partial^{2} u}{\partial x^{2}}+\frac{1}{h^{2}} \frac{\partial^{2} u}{\partial \eta^{2}}\right)-\frac{\sigma B^{2}}{\rho} u-\frac{\mu}{K} u \\
& u \frac{\partial v}{\partial x}+\frac{v}{h} \frac{\partial v}{\partial \eta}=-\frac{1}{\rho h} \frac{\partial p}{\partial \eta}+v\left(\frac{\partial^{2} v}{\partial x^{2}}+\frac{1}{h^{2}} \frac{\partial^{2} v}{\partial \eta^{2}}\right)-\frac{\sigma B^{2}}{\rho} v-\frac{\mu}{K} v
\end{aligned}
$$

where $v$ is the kinematic coefficient of viscosity, $p$ is the pressure, $\rho$ is the density of the fluid. Equations (5) and (6) are known as Navier - Stokes equation of motion for the magnetohydrodynamic flow of viscous fluid.

The boundary conditions are

$$
\begin{aligned}
& u(x, \eta)=0, v(x, \eta)=0, \text { when } \eta=0 \\
& v(x, \eta)=V, \text { when } \eta=1 \\
& u(x, 1)=-\phi \frac{\partial u}{\partial \eta}
\end{aligned}
$$

Equation (7) represents boundary condition with no-slip for the flow of viscous fluid that passes through the solid surface and equation (8) mathematically describes the rate of suction or 
International Journal of Mathematical, Engineering and Management Sciences

Vol. 4, No. 3, 708-728, 2019

https://dx.doi.org/10.33889/IJMEMS.2019.4.3-056

permeability at the permeable wall which is constant along the length of channel. Equation (9) represents the slip boundary condition.

Introducing stream function $\Psi(x, y)$, we have

$u=\frac{\partial \Psi}{\partial y}$ and $v=-\frac{\partial \Psi}{\partial x}$

In dimensionless form, equation (10) becomes

$u=\frac{1}{h} \frac{\partial \Psi}{\partial \eta}$ and $v=-\frac{\partial \Psi}{\partial x}$

We assume the following stream function for the flow as (Terril and Shrestha, 1964)

$\Psi(x, \eta)=(h U-V x) f(\eta)$

where $U$ is the average velocity of the viscous fluid at the entrance of the channel $x=0$.

Using equation (12), the velocity components $u$ and $v$ in equation (11) are given by:

$$
\begin{aligned}
& u=\left[U-\frac{V x}{h}\right] f^{\prime}(\eta), \\
& v=V f(\eta)
\end{aligned}
$$

Eliminating $p$ from equations (5) and (6) and then using equations (10), (11) and (12), we get

$$
\begin{aligned}
& \operatorname{Re}\left[f^{\prime} f^{\prime \prime}-f f^{\prime \prime \prime}-\chi f^{\prime \prime}-r f^{\prime \prime}\right]+f^{i v}=0, \\
& \text { or } \quad \operatorname{Re} \frac{d}{d \eta}\left[f^{\prime 2}-f f^{\prime \prime}-\chi f^{\prime}-r f^{\prime}\right]+f^{i v}=0 .
\end{aligned}
$$

Integrating the above differential equation, we get

$$
\operatorname{Re}\left[f^{\prime 2}-f f^{\prime \prime}-\chi f^{\prime}-r f^{\prime}\right]+f^{\prime \prime \prime}=A
$$

Here $\operatorname{Re}=(V \rho h / \mu)$ represents the Reynolds Number of the suction, $r=(h \mu / K)$ is constant and $K=r \operatorname{Re}$ represents the permeability parameter of the porous medium. We take

$\chi=H_{0}^{2} \mu_{e}^{2} \sigma h / \rho V, \operatorname{Re}=h V / v$ and $v=\mu / \rho, M=\sqrt{ }(\operatorname{Re} . \chi)=B h(\sigma / v \rho)^{1 / 2}$.

The differential equation given by equation (16) is a non-linear differential equation and is solved by using Perturbation method. The boundary conditions on $f(\eta)$ are 
International Journal of Mathematical, Engineering and Management Sciences

Vol. 4, No. 3, 708-728, 2019

https://dx.doi.org/10.33889/IJMEMS.2019.4.3-056

$f_{n}=0$ at $\left.\eta=0, \forall n \geq 0\right\}$,

$f_{n}^{\prime}=0$ at $\left.\eta=0, \forall n \geq 0\right\}$,

$\left.\begin{array}{ll}f_{0}=1, f_{n}=0 & \text { at } \eta=1, \forall n \geq 1 \\ f_{n}^{\prime}+\phi f_{n}^{\prime \prime}=0 & \text { at } \eta=1, \forall n \geq 0\end{array}\right\}$

The process of solving the differential equation given by equation (16) using Perturbation method is given below.

\section{Method of Solution}

The non-linear differential equation given by equation (16) is solved by a perturbation approach subject to the boundary conditions given in equations (18) and (19). However for the special case when Re is multiplied by $\chi$ and $r$, then it will be Re. $\chi=M^{2}$ and Re. $r=K$, where $M$ is Hartmann number and $K$ is permeability parameter. In this situation, we take the following expansions for $f(\eta)$ and $A$ as power series in $\operatorname{Re}$ as

$$
f(\eta)=f_{0}(\eta)+\operatorname{Re} f_{1}(\eta)+\operatorname{Re}^{2} f_{2}(\eta)+\ldots=\sum_{n=0}^{\infty} \operatorname{Re}^{n} f_{n}(\eta)
$$

and $A=A_{0}+A_{1} \operatorname{Re}+A_{2} \operatorname{Re}^{2}+\ldots=\sum_{n=0}^{\infty} A_{n} \operatorname{Re}^{n}$.

Putting these expressions of $f(\eta)$ and $A$ from equations (20) and (21) in equation (16), we get

$$
\begin{aligned}
& \operatorname{Re}\left[\left(f_{0}^{\prime}(\eta)+\operatorname{Re} f_{1}^{\prime}(\eta)+\operatorname{Re}^{2} f_{2}^{\prime}(\eta)+\ldots\right)^{2}\right. \\
& -\left(f_{0}(\eta)+\operatorname{Re} f_{1}(\eta)+\operatorname{Re}^{2} f_{2}(\eta)+\ldots\right) \cdot\left(f_{0}^{\prime \prime}(\eta)+\operatorname{Re} f_{1}^{\prime \prime}(\eta)+\operatorname{Re}^{2} f_{2}^{\prime \prime}(\eta)+\ldots\right) \\
& \left.-\chi\left(f_{0}^{\prime}(\eta)+\operatorname{Re} f_{1}^{\prime}(\eta)+\operatorname{Re}^{2} f_{2}^{\prime}(\eta)+\ldots\right)-r\left(f_{0}^{\prime}(\eta)+\operatorname{Re} f_{1}^{\prime}(\eta)+\operatorname{Re}^{2} f_{2}^{\prime}(\eta)+\ldots\right)\right] \\
& +f_{0}^{\prime \prime \prime}(\eta)+\operatorname{Re} f_{1}^{\prime \prime \prime}(\eta)+\operatorname{Re}^{2} f_{2}^{\prime \prime \prime}(\eta)+\ldots=A_{0}+A_{1} \operatorname{Re}+A_{2} \operatorname{Re}^{2}+\ldots
\end{aligned}
$$

For first approximation, we take the coefficient of $\operatorname{Re}^{0}, \operatorname{Re}$ and $\operatorname{Re}^{2}$ from equation (22). Then we have

$$
\begin{aligned}
& f_{0}^{\prime \prime \prime}=A_{0}, \\
& f_{1}^{\prime \prime \prime}=A_{1}+f_{0} f_{0}^{\prime \prime}-f_{0}^{\prime 2}+\chi f_{0}^{\prime}+r f_{0}^{\prime}, \\
& f_{2}^{\prime \prime \prime}=A_{2}+f_{0} f_{1}^{\prime \prime}+f_{1} f_{0}^{\prime \prime}-2 f_{0}^{\prime} f_{1}^{\prime}+\chi f_{1}^{\prime}+r f_{1}^{\prime},
\end{aligned}
$$

Integrating and solving equations (23), (24) and (25) under the boundary conditions given in equations (18) and (19), we get

$$
f_{0}=-\frac{2(1+\phi)}{(1+4 \phi)} \eta^{3}+\frac{3(1+2 \phi)}{(1+4 \phi)} \eta^{2}
$$


International Journal of Mathematical, Engineering and Management Sciences

Vol. 4, No. 3, 708-728, 2019

https://dx.doi.org/10.33889/IJMEMS.2019.4.3-056

$$
\begin{aligned}
& f_{1}=-\frac{2(1+\phi)^{2}}{35(1+4 \phi)^{2}} \eta^{7}+\frac{(1+\phi)(1+2 \phi)}{5(1+4 \phi)^{2}} \eta^{6}-\frac{3(1+2 \phi)^{2}}{10(1+4 \phi)^{2}} \eta^{5} \\
& +\frac{\left(27+270 \phi+808 \phi^{2}+880 \phi^{3}\right)}{70(1+4 \phi)^{3}} \eta^{3}-\frac{\left(8+88 \phi+272 \phi^{2}+312 \phi^{3}\right)}{35(1+4 \phi)^{3}} \eta^{2} \\
& +\chi\left[-\frac{(1+\phi)}{10(1+4 \phi)} \eta^{5}+\frac{(1+2 \phi)}{4(1+4 \phi)} \eta^{4}-\frac{\left(3+21 \phi+48 \phi^{2}\right)}{15(1+4 \phi)^{2}} \eta^{3}+\frac{\left(1+8 \phi+32 \phi^{2}\right)}{20(1+4 \phi)^{2}} \eta^{2}\right] \\
& +r\left[-\frac{(1+\phi)}{10(1+4 \phi)} \eta^{5}+\frac{(1+2 \phi)}{4(1+4 \phi)} \eta^{4}-\frac{\left(3+21 \phi+48 \phi^{2}\right)}{15(1+4 \phi)^{2}} \eta^{3}+\frac{\left(1+8 \phi+32 \phi^{2}\right)}{20(1+4 \phi)^{2}} \eta^{2}\right], \\
& f_{2}=\frac{24(1+\phi)^{3}}{34650(1+4 \phi)^{3}} \eta^{11}-\frac{96(1+\phi)^{2}(1+2 \phi)}{25200(1+4 \phi)^{3}} \eta^{10}+\frac{24(1+\phi)(1+2 \phi)^{2}}{5040(1+4 \phi)^{3}} \eta^{9} \\
& -\frac{18(1+2 \phi)^{3}}{3360(1+4 \phi)^{3}} \eta^{8}+\frac{6(1+\phi)\left(27+270 \phi+808 \phi^{2}+808 \phi^{3}\right)}{7350(1+4 \phi)^{4}} \eta^{7} \\
& -\frac{\left(226+2226 \phi+10968 \phi^{2}+162208 \phi^{3}+13056 \phi^{4}\right)}{4200(1+4 \phi)^{4}} \eta^{6} \\
& +\frac{(1+2 \phi)\left(8+88 \phi+272 \phi^{2}+312 \phi^{3}\right)}{175(1+4 \phi)^{4}} \eta^{5}+\left[\frac{925691+10148227 \phi+5667543 \phi^{2}}{323400(1+4 \phi)^{5}}\right. \\
& \left.+\frac{7425066092 \phi^{3}+133792876 \not \phi^{4}+101877040 \phi^{5}}{323400(1+4 \phi)^{5}}\right] \eta^{3}-\left[\frac{1858001+20497820 \phi}{646800(1+4 \phi)^{5}}\right. \\
& \left.+\frac{114502856 \phi^{2}+14830885430 \phi^{3}+2590851280 \phi^{4}+202973760 \phi^{5}}{646800(1+4 \phi)^{5}}\right] \eta^{2} \\
& +\chi\left[-\frac{12(1+\phi)^{2}}{5040(1+4 \phi)^{2}} \eta^{9}+\frac{36(1+\phi)(1+2 \phi)}{3360(1+4 \phi)^{2}} \eta^{8}-\frac{18\left(3+24 \phi+64 \phi^{2}+48 \phi^{3}\right)}{2100(1+4 \phi)^{3}} \eta^{7}\right. \\
& +\frac{\left(29+261 \phi+920 \phi^{2}+928 \phi^{3}\right)}{1200(1+4 \phi)^{3}} \eta^{6}+\frac{\left(39+390 \phi+408 \phi^{2}-48 \phi^{3}\right)}{4200(1+4 \phi)^{3}} \eta^{5} \\
& -\frac{\left(8+88 \phi+272 \phi^{2}+312 \phi^{3}\right)}{420(1+4 \phi)^{3}} \eta^{4}+\left\{\frac{10280+395600 \phi+4201904 \phi^{2}+1315562 \phi^{3}}{235200(1+4 \phi)^{4}}\right. \\
& \left.+\frac{\left.16259097 \phi^{4}\right)}{235200(1+4 \phi)^{4}}\right\} \eta^{3} \\
& \left.+\frac{\left(2956+386052 \phi+4337504 \phi^{2}+1615210 \phi^{3}+1655196 \not \phi^{4}\right)}{235200(1+4 \phi)^{4}} \eta^{2}\right] \\
& +\chi^{2}\left[-\frac{5(1+\phi)}{2100(1+4 \phi)} \eta^{7}+\frac{4(1+2 \phi)}{600(1+4 \phi)} \eta^{6}-\frac{3\left(3+21 \phi+48 \phi^{2}\right)}{900(1+4 \phi)^{2}} \eta^{5}+\frac{\left(1+8 \phi+32 \phi^{2}\right)}{240(1+4 \phi)^{2}} \eta^{4}\right.
\end{aligned}
$$


International Journal of Mathematical, Engineering and Management Sciences

Vol. 4, No. 3, 708-728, 2019

https://dx.doi.org/10.33889/IJMEMS.2019.4.3-056

$$
\begin{aligned}
+ & \left.\frac{\left(29+377 \phi+1388 \phi^{2}+1824 \phi^{3}\right)}{4200(1+4 \phi)^{3}} \eta^{3}+\frac{\left(71+878 \phi+2920 \phi^{2}+3072 \phi^{3}\right)}{8400(1+4 \phi)^{3}} \eta^{2}\right] \\
& +r\left[-\frac{12(1+\phi)^{2}}{5040(1+4 \phi)^{2}} \eta^{9}+\frac{36(1+\phi)(1+2 \phi)}{3360(1+4 \phi)^{2}} \eta^{8}-\frac{18\left(3+24 \phi+64 \phi^{2}+48 \phi^{3}\right)}{2100(1+4 \phi)^{3}} \eta^{7}\right. \\
& +\frac{\left(29+261 \phi+920 \phi^{2}+928 \phi^{3}\right)}{1200(1+4 \phi)^{3}} \eta^{6}+\frac{\left(39+390 \phi+408 \phi^{2}-48 \phi^{3}\right)}{4200(1+4 \phi)^{3}} \eta^{5} \\
& -\frac{\left(8+88 \phi+272 \phi^{2}+312 \phi^{3}\right)}{420(1+4 \phi)^{3}} \eta^{4}+\left\{\frac{10280+395600 \phi+4201904 \phi^{2}+1315562 \phi^{3}}{235200(1+4 \phi)^{4}}\right. \\
& \left.\left.+\frac{16259097 \phi^{4}}{235200(1+4 \phi)^{4}}\right\} \eta^{3}+\frac{\left(2956+386052 \phi+4337504 \phi^{2}+1615210 \phi^{3}+16551961 \phi^{4}\right)}{235200(1+4 \phi)^{4}} \eta^{2}\right] \\
& +r^{2}\left[-\frac{5(1+\phi)}{2100(1+4 \phi)} \eta^{7}+\frac{4(1+2 \phi)}{600(1+4 \phi)} \eta^{6}-\frac{3\left(3+21 \phi+48 \phi^{2}\right)}{900(1+4 \phi)^{2}} \eta^{5}+\frac{\left(1+8 \phi+32 \phi^{2}\right)}{240(1+4 \phi)^{2}} \eta^{4}\right. \\
& \left.+\frac{\left(29+377 \phi+1388 \phi^{2}+1824 \phi^{3}\right)}{4200(1+4 \phi)^{3}} \eta^{3}+\frac{\left(71+878 \phi+2920 \phi^{2}+3072 \phi^{3}\right)}{8400(1+4 \phi)^{3}} \eta^{2}\right],
\end{aligned}
$$

Substituting $f_{0}, f_{1}$ and $f_{2}$ in equation (20), we get

$$
\begin{aligned}
f(\eta)= & -\frac{2(1+\phi)}{(1+4 \phi)} \eta^{3}+\frac{3(1+2 \phi)}{(1+4 \phi)} \eta^{2}+\operatorname{Re}\left[-\frac{2(1+\phi)^{2}}{(1+4 \phi)^{2}} \eta^{7}+\frac{(1+\phi)(1+2 \phi)}{5(1+4 \phi)^{2}} \eta^{6}\right. \\
& \left.-\frac{3(1+2 \phi)^{2}}{10(1+4 \phi)^{2}} \eta^{5}+\frac{\left(27+270 \phi+808 \phi^{2}+880 \phi^{3}\right)}{70(1+4 \phi)^{3}} \eta^{3}-\frac{\left(8+88 \phi+272 \phi^{2}+312 \phi^{3}\right)}{35(1+4 \phi)^{3}} \eta^{2}\right] \\
& +\operatorname{Re} \cdot \chi\left[-\frac{(1+\phi)}{10(1+4 \phi)} \eta^{5}+\frac{(1+2 \phi)}{4(1+4 \phi)} \eta^{4}-\frac{\left(3+21 \phi+48 \phi^{2}\right)}{15(1+4 \phi)^{2}} \eta^{3}+\frac{\left(1+8 \phi+32 \phi^{2}\right)}{20(1+4 \phi)^{2}} \eta^{2}\right] \\
& +\operatorname{Re} \cdot r\left[-\frac{(1+\phi)}{10(1+4 \phi)} \eta^{5}+\frac{(1+2 \phi)}{4(1+4 \phi)} \eta^{4}-\frac{\left(3+21 \phi+48 \phi^{2}\right)}{15(1+4 \phi)^{2}} \eta^{3}+\frac{\left(1+8 \phi+32 \phi^{2}\right)}{20(1+4 \phi)^{2}} \eta^{2}\right] \\
& +\operatorname{Re}\left[\frac{24(1+\phi)^{3}}{34650(1+4 \phi)^{3}} \eta^{11}-\frac{96(1+\phi)^{2}(1+2 \phi)}{25200(1+4 \phi)^{3}} \eta^{10}+\frac{24(1+\phi)(1+2 \phi)^{2}}{5040(1+4 \phi)^{3}} \eta^{9}\right. \\
& -\frac{18(1+2 \phi)^{3}}{3360(1+4 \phi)^{3}} \eta^{8}+\frac{6(1+\phi)\left(27+270 \phi+808 \phi^{2}+880 \phi^{3}\right)}{7350(1+4 \phi)^{4}} \eta^{7} \\
& -\frac{\left(226+2226 \phi+10968 \phi^{2}+162208 \phi^{3}+13056 \phi^{4}\right)}{4200(1+4 \phi)^{4}} \eta^{6}
\end{aligned}
$$


International Journal of Mathematical, Engineering and Management Sciences

Vol. 4, No. 3, 708-728, 2019

https://dx.doi.org/10.33889/IJMEMS.2019.4.3-056

$$
\begin{aligned}
& +\frac{(1+2 \phi)\left(8+88 \phi+272 \phi^{2}+312 \phi^{3}\right)}{175(1+4 \phi)^{4}} \eta^{5}+\left\{\frac{925691+10148227 \phi+5667543 \phi^{2}}{323400(1+4 \phi)^{5}}\right. \\
& \left.+\frac{7425066092 \phi^{3}+133792876 \not \phi^{4}+101877040 \phi^{5}}{323400(1+4 \phi)^{5}}\right\} \eta^{3}-\left\{\frac{1858001+20497820 \phi}{646800(1+4 \phi)^{5}}\right. \\
& \left.\left.+\frac{114502856 \phi^{2}+14830885430 \phi^{3}+2590851280 \phi^{4}+202973760 \phi^{5}}{646800(1+4 \phi)^{5}}\right\} \eta^{2}\right] \\
& +\operatorname{Re}^{2} \chi\left[-\frac{12(1+\phi)^{2}}{5040(1+4 \phi)^{2}} \eta^{9}+\frac{36(1+\phi)(1+2 \phi)}{3360(1+4 \phi)^{2}} \eta^{8}-\frac{18\left(3+24 \phi+64 \phi^{2}+48 \phi^{3}\right)}{2100(1+4 \phi)^{3}} \eta^{7}\right. \\
& +\frac{\left(29+261 \phi+920 \phi^{2}+928 \phi^{3}\right)}{1200(1+4 \phi)^{3}} \eta^{6}+\frac{\left(39+390 \phi+408 \phi^{2}-48 \phi^{3}\right)}{4200(1+4 \phi)^{3}} \eta^{5} \\
& -\frac{\left(8+88 \phi+272 \phi^{2}+312 \phi^{3}\right)}{420(1+4 \phi)^{3}} \eta^{4}+\left\{\frac{10280+395600 \phi+42019004 \phi^{2}+1315562 \phi^{3}}{235200(1+4 \phi)^{4}}\right. \\
& \left.+\frac{\left.16259097 \phi^{4}\right)}{235200(1+4 \phi)^{4}}\right\} \eta^{3}+\left\{\frac{2956+386052 \phi+4337504 \phi^{2}+1615210 \phi^{3}}{235200(1+4 \phi)^{4}}\right. \\
& \left.\left.+\frac{16551961 \phi^{4}}{235200(1+4 \phi)^{4}}\right\} \eta^{2}\right]+\operatorname{Re}^{2} \chi^{2}\left[-\frac{5(1+\phi)}{2100(1+4 \phi)} \eta^{7}+\frac{4(1+2 \phi)}{600(1+4 \phi)} \eta^{6}\right. \\
& -\frac{3\left(3+21 \phi+48 \phi^{2}\right)}{900(1+4 \phi)^{2}} \eta^{5}+\frac{\left(1+8 \phi+32 \phi^{2}\right)}{240(1+4 \phi)^{2}} \eta^{4}+\frac{\left(29+377 \phi+1388 \phi^{2}+1824 \phi^{3}\right)}{4200(1+4 \phi)^{3}} \eta^{3} \\
& \left.+\frac{\left(71+878 \phi+2920 \phi^{2}+3072 \phi^{3}\right)}{8400(1+4 \phi)^{3}} \eta^{2}\right]+\operatorname{Re}^{2} r\left[-\frac{12(1+\phi)^{2}}{5040(1+4 \phi)^{2}} \eta^{9}\right. \\
& +\frac{36(1+\phi)(1+2 \phi)}{3360(1+4 \phi)^{2}} \eta^{8}-\frac{18\left(3+24 \phi+64 \phi^{2}+48 \phi^{3}\right)}{2100(1+4 \phi)^{3}} \eta^{7} \\
& +\frac{\left(29+261 \phi+920 \phi^{2}+928 \phi^{3}\right)}{1200(1+4 \phi)^{3}} \eta^{6}+\frac{\left(39+390 \phi+408 \phi^{2}-48 \phi^{3}\right)}{4200(1+4 \phi)^{3}} \eta^{5} \\
& -\frac{\left(8+88 \phi+272 \phi^{2}+312 \phi^{3}\right)}{420(1+4 \phi)^{3}} \eta^{4} \\
& +\frac{\left(10280+395600 \phi+4201904 \phi^{2}+1315562 \phi^{3}+16259097 \phi^{4}\right)}{235200(1+4 \phi)^{4}} \eta^{3} \\
& +\frac{\left(2956+386052 \phi \phi+4337504 \phi^{2}+1615210 \phi^{3}+1655196 \not \phi^{4}\right)}{235200(1+4 \phi)^{4}} \eta^{2}
\end{aligned}
$$


International Journal of Mathematical, Engineering and Management Sciences

Vol. 4, No. 3, 708-728, 2019

https://dx.doi.org/10.33889/IJMEMS.2019.4.3-056

$$
\begin{aligned}
& +\operatorname{Re}^{2} r^{2}\left[-\frac{5(1+\phi)}{2100(1+4 \phi)} \eta^{7}+\frac{4(1+2 \phi)}{600(1+4 \phi)} \eta^{6}-\frac{3\left(3+21 \phi+48 \phi^{2}\right)}{900(1+4 \phi)^{2}} \eta^{5}\right. \\
& +\frac{\left(1+8 \phi+32 \phi^{2}\right)}{240(1+4 \phi)^{2}} \eta^{4}+\frac{\left(29+377 \phi+1388 \phi^{2}+1824 \phi^{3}\right)}{4200(1+4 \phi)^{3}} \eta^{3} \\
& \left.+\frac{\left(71+878 \phi+2920 \phi^{2}+3072 \phi^{3}\right)}{8400(1+4 \phi)^{3}} \eta^{2}\right]+\ldots
\end{aligned}
$$

or $f(\eta)=-\frac{2(1+\phi)}{(1+4 \phi)} \eta^{3}+\frac{3(1+2 \phi)}{(1+4 \phi)} \eta^{2}+\operatorname{Re}\left[-\frac{2(1+\phi)^{2}}{(1+4 \phi)^{2}} \eta^{7}+\frac{(1+\phi)(1+2 \phi)}{5(1+4 \phi)^{2}} \eta^{6}\right.$

$$
\begin{aligned}
& \left.-\frac{3(1+2 \phi)^{2}}{10(1+4 \phi)^{2}} \eta^{5}+\frac{\left(27+270 \phi+808 \phi^{2}+880 \phi^{3}\right)}{70(1+4 \phi)^{3}} \eta^{3}-\frac{\left(8+88 \phi+272 \phi^{2}+312 \phi^{3}\right)}{35(1+4 \phi)^{3}} \eta^{2}\right] \\
& +M^{2}\left[-\frac{(1+\phi)}{10(1+4 \phi)} \eta^{5}+\frac{(1+2 \phi)}{4(1+4 \phi)} \eta^{4}-\frac{\left(3+21 \phi+48 \phi^{2}\right)}{15(1+4 \phi)^{2}} \eta^{3}+\frac{\left(1+8 \phi+32 \phi^{2}\right)}{20(1+4 \phi)^{2}} \eta^{2}\right] \\
& +K\left[-\frac{(1+\phi)}{10(1+4 \phi)} \eta^{5}+\frac{(1+2 \phi)}{4(1+4 \phi)} \eta^{4}-\frac{\left(3+21 \phi+48 \phi^{2}\right)}{15(1+4 \phi)^{2}} \eta^{3}+\frac{\left(1+8 \phi+32 \phi^{2}\right)}{20(1+4 \phi)^{2}} \eta^{2}\right] \\
& +\operatorname{Re}^{2}\left[\frac{24(1+\phi)^{3}}{34650(1+4 \phi)^{3}} \eta^{11}-\frac{96(1+\phi)^{2}(1+2 \phi)}{25200(1+4 \phi)^{3}} \eta^{10}+\frac{24(1+\phi)(1+2 \phi)^{2}}{5040(1+4 \phi)^{3}} \eta^{9}\right. \\
& -\frac{18(1+2 \phi)^{3}}{3360(1+4 \phi)^{3}} \eta^{8}+\frac{6(1+\phi)\left(27+270 \phi+808 \phi^{2}+880 \phi^{3}\right)}{7350(1+4 \phi)^{4}} \eta^{7} \\
& -\frac{\left(226+2226 \phi+10968 \phi^{2}+162208 \phi^{3}+13056 \phi^{4}\right)}{4200(1+4 \phi)^{4}} \eta^{6} \\
& +\frac{(1+2 \phi)\left(8+88 \phi+272 \phi^{2}+312 \phi^{3}\right)}{175(1+4 \phi)^{4}} \eta^{5}+\left\{\frac{925691+10148227 \phi+5667543 \phi^{2}}{323400(1+4 \phi)^{5}}\right. \\
& \left.+\frac{7425066092 \phi^{3}+1337928760 \phi^{4}+10187704 \phi \phi^{5}}{323400(1+4 \phi)^{5}}\right\} \eta^{3}-\left\{\frac{1858001+20497820 \phi}{646800(1+4 \phi)^{5}}\right. \\
& \left.\left.+\frac{114502856 \phi^{2}+14830885430 \phi^{3}+2590851280 \phi^{4}+202973760 \phi^{5}}{646800(1+4 \phi)^{5}}\right\} \eta^{2}\right] \\
& +M^{2} \operatorname{Re}\left[-\frac{12(1+\phi)^{2}}{5040(1+4 \phi)^{2}} \eta^{9}+\frac{36(1+\phi)(1+2 \phi)}{3360(1+4 \phi)^{2}} \eta^{8}-\frac{18\left(3+24 \phi+64 \phi^{2}+48 \phi^{3}\right)}{2100(1+4 \phi)^{3}} \eta^{7}\right. \\
& +\frac{\left(29+261 \phi+920 \phi^{2}+928 \phi^{3}\right)}{1200(1+4 \phi)^{3}} \eta^{6}+\frac{\left(39+390 \phi+408 \phi^{2}-48 \phi^{3}\right)}{4200(1+4 \phi)^{3}} \eta^{5}
\end{aligned}
$$


International Journal of Mathematical, Engineering and Management Sciences

Vol. 4, No. 3, 708-728, 2019

https://dx.doi.org/10.33889/IJMEMS.2019.4.3-056

$$
\begin{aligned}
& -\frac{\left(8+88 \phi+272 \phi^{2}+312 \phi^{3}\right)}{420(1+4 \phi)^{3}} \eta^{4}+\left\{\frac{10280+395600 \phi+4201904 \phi^{2}+1315562 \phi^{3}}{235200(1+4 \phi)^{4}}\right. \\
& \left.\left.+\frac{16259097 \phi^{4}}{235200(1+4 \phi)^{4}}\right\} \eta^{3}+\frac{\left(2956+386052 \phi+4337504 \phi^{2}+1615210 \phi^{3}+1655196 \phi^{4}\right)}{235200(1+4 \phi)^{4}} \eta^{2}\right] \\
& +M^{4}\left[-\frac{5(1+\phi)}{2100(1+4 \phi)} \eta^{7}+\frac{4(1+2 \phi)}{600(1+4 \phi)} \eta^{6}-\frac{3\left(3+21 \phi+48 \phi^{2}\right)}{900(1+4 \phi)^{2}} \eta^{5}+\frac{\left(1+8 \phi+32 \phi^{2}\right)}{240(1+4 \phi)^{2}} \eta^{4}\right. \\
& \left.+\frac{\left(29+377 \phi+1388 \phi^{2}+1824 \phi^{3}\right)}{4200(1+4 \phi)^{3}} \eta^{3}+\frac{\left(71+878 \phi+2920 \phi^{2}+3072 \phi^{3}\right)}{8400(1+4 \phi)^{3}} \eta^{2}\right] \\
& +\operatorname{Re} K\left[-\frac{12(1+\phi)^{2}}{5040(1+4 \phi)^{2}} \eta^{9}+\frac{36(1+\phi)(1+2 \phi)}{3360(1+4 \phi)^{2}} \eta^{8}-\frac{18\left(3+24 \phi+64 \phi^{2}+48 \phi^{3}\right)}{2100(1+4 \phi)^{3}} \eta^{7}\right. \\
& +\frac{\left(29+261 \phi+920 \phi^{2}+928 \phi^{3}\right)}{1200(1+4 \phi)^{3}} \eta^{6}+\frac{\left(39+390 \phi+408 \phi^{2}-48 \phi^{3}\right)}{4200(1+4 \phi)^{3}} \eta^{5} \\
& -\frac{\left(8+88 \phi+272 \phi^{2}+312 \phi^{3}\right)}{420(1+4 \phi)^{3}} \eta^{4}+\left\{\frac{10280+395600 \phi+4201904 \phi^{2}+1315562 \phi^{3}}{235200(1+4 \phi)^{4}}\right. \\
& \left.+\frac{16259097 \phi^{4}}{235200(1+4 \phi)^{4}}\right\} \eta^{3}+\frac{\left(2956+386052 \phi+4337504 \phi^{2}+1615210 \phi^{3}+16551961 \phi^{4}\right)}{235200(1+4 \phi)^{4}} \eta^{2} \\
& +K^{2}\left[-\frac{5(1+\phi)}{2100(1+4 \phi)} \eta^{7}+\frac{4(1+2 \phi)}{600(1+4 \phi)} \eta^{6}-\frac{3\left(3+21 \phi+48 \phi^{2}\right)}{900(1+4 \phi)^{2}} \eta^{5}\right. \\
& +\frac{\left(1+8 \phi+32 \phi^{2}\right)}{240(1+4 \phi)^{2}} \eta^{4}+\frac{\left(29+377 \phi+1388 \phi^{2}+1824 \phi^{3}\right)}{4200(1+4 \phi)^{3}} \eta^{3} \\
& \left.+878 \phi+2920 \phi^{2}+3072 \phi^{3}\right) \\
& +2 \\
& +
\end{aligned}
$$

Now putting $M=0, K=0$ in equation (30), we get the solution which matches with Bujurke et al. (2010) and is given below

$$
\begin{aligned}
f(\eta) & =-\frac{2(1+\phi)}{(1+4 \phi)} \eta^{3}+\frac{3(1+2 \phi)}{(1+4 \phi)} \eta^{2}+\operatorname{Re}\left[-\frac{2(1+\phi)^{2}}{(1+4 \phi)^{2}} \eta^{7}+\frac{(1+\phi)(1+2 \phi)}{5(1+4 \phi)^{2}} \eta^{6}\right. \\
& \left.-\frac{3(1+2 \phi)^{2}}{10(1+4 \phi)^{2}} \eta^{5}+\frac{\left(27+270 \phi+808 \phi^{2}+880 \phi^{3}\right)}{70(1+4 \phi)^{3}} \eta^{3}-\frac{\left(8+88 \phi+272 \phi^{2}+312 \phi^{3}\right)}{35(1+4 \phi)^{3}} \eta^{2}\right] \\
& +\operatorname{Re}^{2}\left[\frac{24(1+\phi)^{3}}{34650(1+4 \phi)^{3}} \eta^{11}-\frac{96(1+\phi)^{2}(1+2 \phi)}{25200(1+4 \phi)^{3}} \eta^{10}+\frac{24(1+\phi)(1+2 \phi)^{2}}{5040(1+4 \phi)^{3}} \eta^{9}\right.
\end{aligned}
$$


International Journal of Mathematical, Engineering and Management Sciences

Vol. 4, No. 3, 708-728, 2019

https://dx.doi.org/10.33889/IJMEMS.2019.4.3-056

$$
\begin{aligned}
& -\frac{18(1+2 \phi)^{3}}{3360(1+4 \phi)^{3}} \eta^{8}+\frac{6(1+\phi)\left(27+270 \phi+808 \phi^{2}+880 \phi^{3}\right)}{7350(1+4 \phi)^{4}} \eta^{7}-\left\{\frac{226+2226 \phi}{4200(1+4 \phi)^{4}}\right. \\
& \left.+\frac{10968 \phi^{2}+162208 \phi^{3}+13056 \phi^{4}}{4200(1+4 \phi)^{4}}\right\} \eta^{6}+\frac{(1+2 \phi)\left(8+88 \phi+272 \phi^{2}+312 \phi^{3}\right)}{175(1+4 \phi)^{4}} \eta^{5} \\
& +\left\{\frac{925691+10148227 \phi+56675432 \phi^{2}+742506609 \not \phi^{3}+133792876 \phi^{4}}{323400(1+4 \phi)^{5}}\right. \\
& \left.+\frac{10187704 \phi \phi^{5}}{323400(1+4 \phi)^{5}}\right\} \eta^{3}-\left\{\frac{1858001+20497820 \phi+114502856 \phi^{2}}{646800(1+4 \phi)^{5}}\right. \\
& \left.\left.+\frac{14830885430 \phi^{3}+2590851280 \phi^{4}+202973760 \phi^{5}}{646800(1+4 \phi)^{5}}\right\} \eta^{2}\right]+\ldots
\end{aligned}
$$

\section{Results and Discussion}

In order to get a physical significance of this problem, graphs are plotted with the help of MATLAB software by giving numerical values to various parameters which are present in the mathematical formulation of the problem. The velocity of the viscous fluid has been drawn for different values of magnetic parameter $M$ (Hartmann Number), permeability parameter $K$, Reynolds number Re, slip coefficient $\phi$ and the width of the channel $h$. The objective of the present study is to investigate the effects of a magnetic field and the permeability parameter on the flow of a viscous fluid in a channel with a porous bounding wall of different permeabilities. The graph of the velocity against distance from the non-porous wall has been plotted in Figure 1 by taking constant values of slip coefficient, Reynolds number, permeability parameter and different values of the magnetic parameter. In the presence of magnetic field $M=1.0$, the velocity of fluid slightly increases and then decreases with the increase of the distance from the nonporous wall. The velocity of the fluid increases sharply with the increase of the magnetic field $(M$ $=1.0,3.0,5.0,7.0)$.

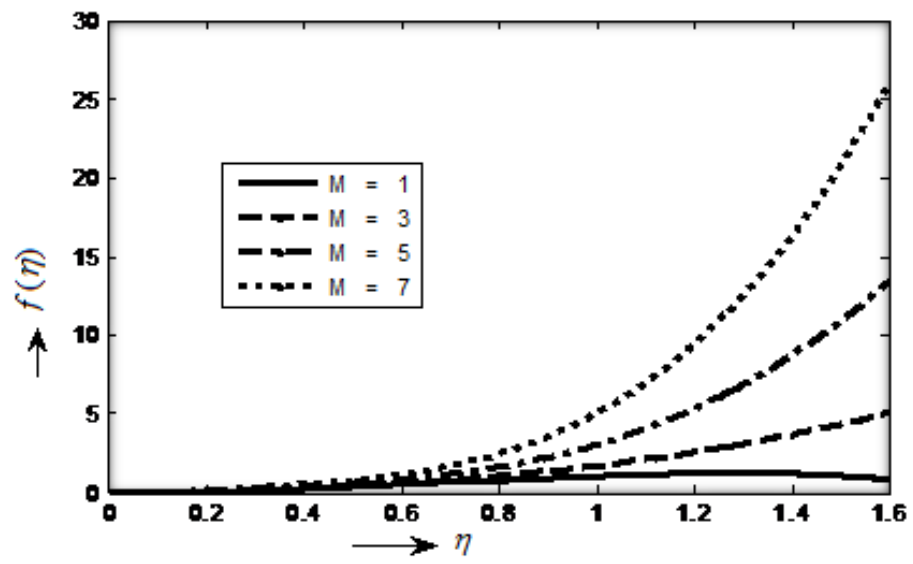

Figure 1. The graph of fluid velocity $f(\eta)$ against $\eta$ for magnetic parameters $(M=1.0,3.0,5.0,7.0)$ by taking parameters $K=0.8, \operatorname{Re}=2.0$ and $\phi=0.5$ 
International Journal of Mathematical, Engineering and Management Sciences

Vol. 4, No. 3, 708-728, 2019

https://dx.doi.org/10.33889/IJMEMS.2019.4.3-056

In Figure 2, the graph of $f(\eta)$ is plotted against $\eta$ by taking different values of permeability parameter $K$ for constant parameters $M$, Re and $\phi$. The graph shows that the velocity of the fluid decreases slowly with the increase of permeability parameter porous medium. The velocity of the fluid against the distance from the non-porous wall has been plotted in Figure 3 for different values of the Reynolds number by taking $M=2, K=0.8$ and $\phi=0.3$. This graph reveals that the velocity of the fluid decreases with the increase of the Reynolds number. Figures (4) and (5) show that the velocity of the fluid decreases with the increase of Reynolds number Re and slip coefficient $\phi$ for constant values of magnetic and permeability parameters. These graphs also reveal that the velocity of the fluid decreases as the distance from the non-porous wall increases.

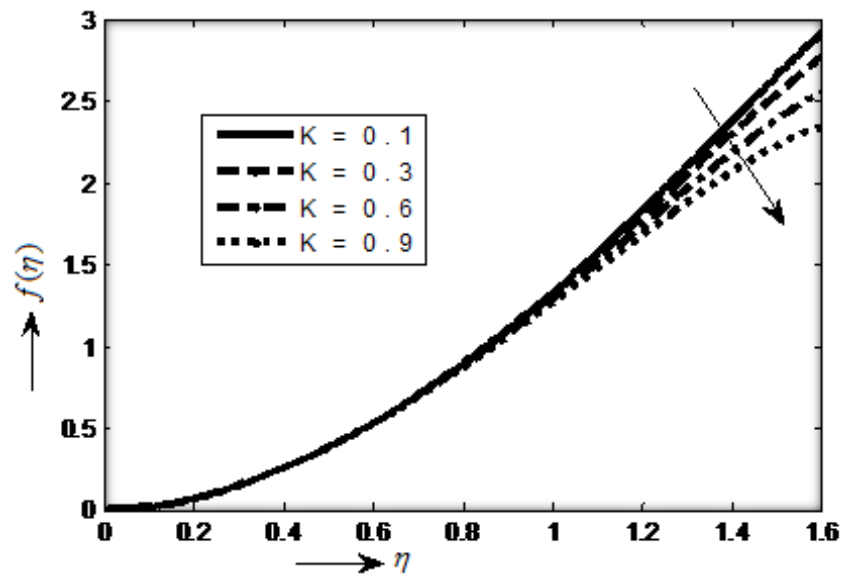

Figure 2. Variation of fluid velocity $f(\eta)$ against $\eta$ for permeability parameters $K(0.1,0.3,0.6,0.9)$ by taking parameters $M=2, \operatorname{Re}=2.0$ and $\phi=0.5$

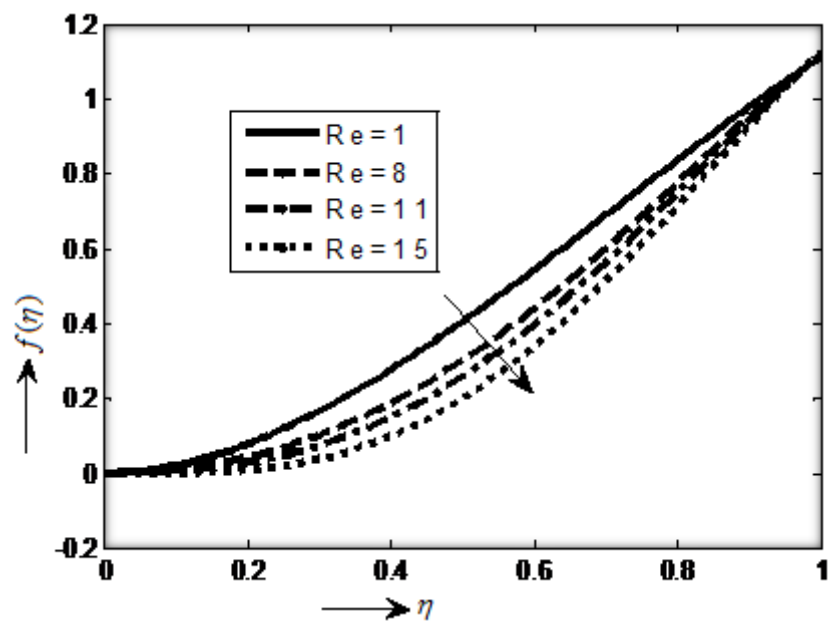

Figure 3.Variation of fluid velocity $f(\eta)$ against $\eta$ for Reynolds Number $\operatorname{Re}(1,8,11,15)$ by taking parameters $M=2, K=0.8$ and $\phi=0.3$ 
International Journal of Mathematical, Engineering and Management Sciences

Vol. 4, No. 3, 708-728, 2019

https://dx.doi.org/10.33889/IJMEMS.2019.4.3-056

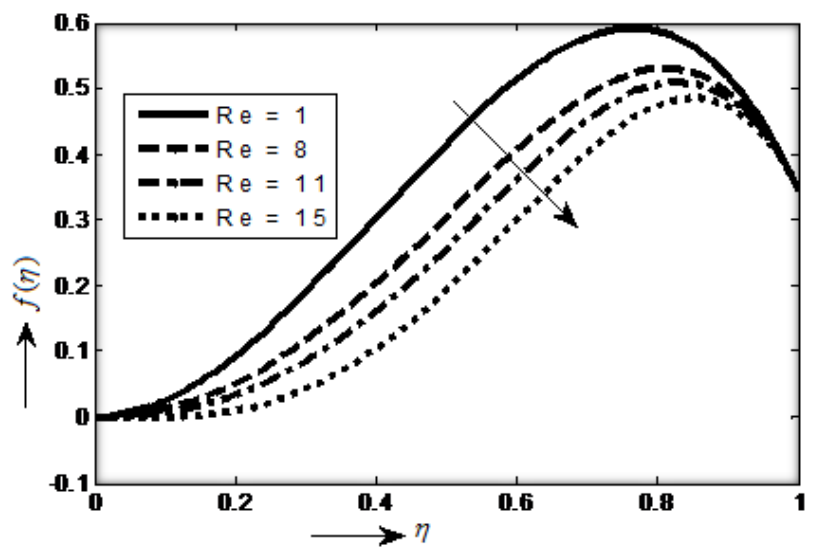

Figure 4. Variation of fluid velocity $f(\eta)$ with respect to $\eta$ for Reynolds Numbers $\operatorname{Re}(1,8,11,15)$ by taking parameters $M=2, K=0.8$ and $\phi=0.05$

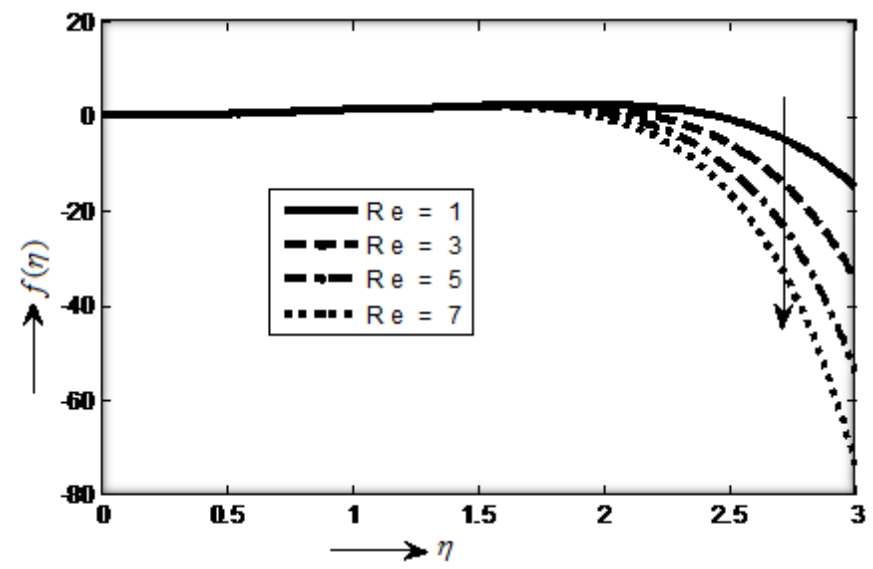

Figure 5. Variation of fluid velocity $f(\eta)$ with respect to $\eta$ for Reynolds Numbers $\operatorname{Re}(1,3,5,7)$ by taking parameters $M=2, K=0.8$ and $\phi=0.5$

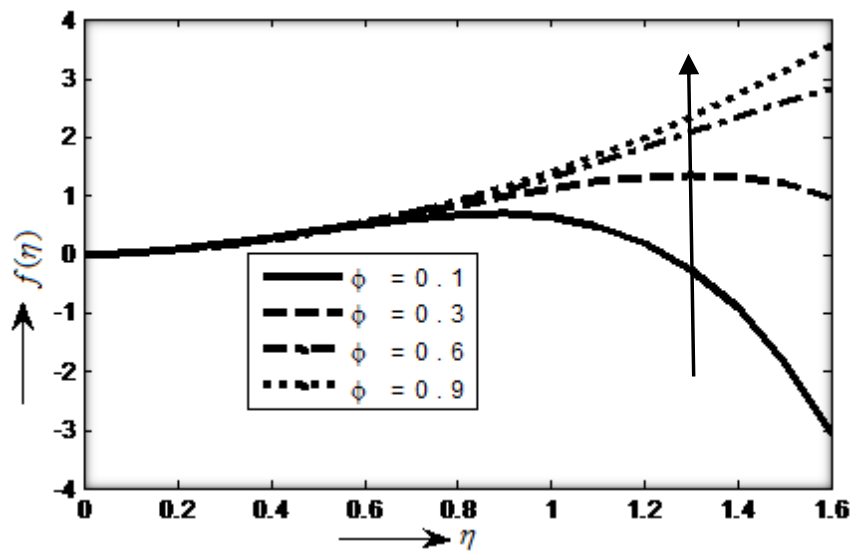

Figure 6. Variation of fluid velocity $f(\eta)$ against $\eta$ for slip coefficients $\phi(0.1,0.3,0.6,0.9)$ by taking parameters $M=2, K=0.8$ and $\operatorname{Re}=2$ 
International Journal of Mathematical, Engineering and Management Sciences

Vol. 4, No. 3, 708-728, 2019

https://dx.doi.org/10.33889/IJMEMS.2019.4.3-056

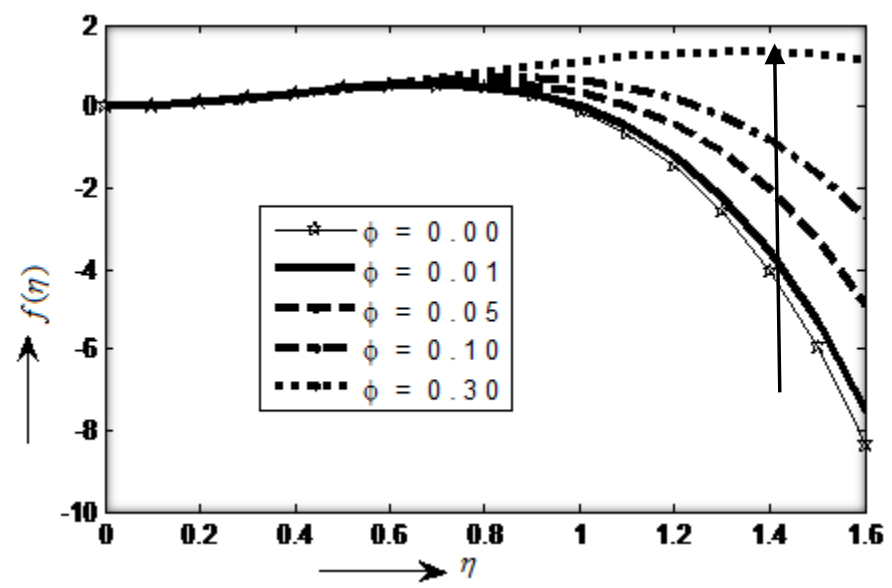

Figure 7. Variation of fluid velocity $f(\eta)$ against $\eta$ for slip coefficients $\phi(0.0,0.01,0.05,0.10,0.30)$ by taking parameters $M=2, K=0.8$ and $\mathrm{Re}=0.1$

In Figures 6 and 7, the velocity of the fluid has been plotted against the distance from the nonporous wall for constant parameters $M, K$ and Re. These graphs show that with the increase of slip coefficient $\phi$, the velocity of the fluid increases sharply but the velocity of the fluid decreases as the distance from the non-porous wall increases.

Figure 8 shows the variation of the velocity of the fluid against the slip coefficient $\phi$ by taking different values of magnetic parameter $M$ for constant values of parameters $\operatorname{Re}, K$ and $\eta$ and it has been found that with the increase of magnetic parameter, the velocity of fluid increases sharply.

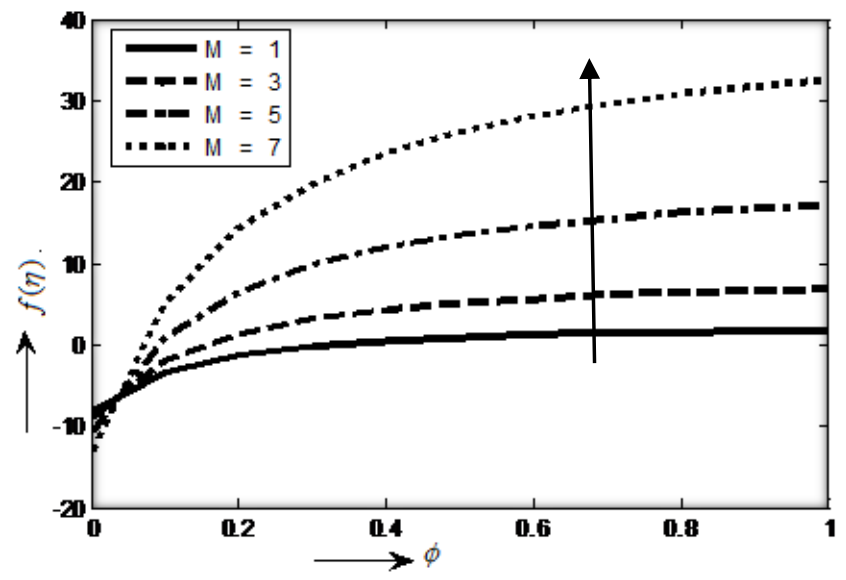

Figure 8. Variation of fluid velocity $f(\eta)$ against $\phi$ for Magnetic parameters $M(1,3,5,7)$ by taking parameters $\eta=1.6, K=0.8$ and $\operatorname{Re}=0.5$ 
International Journal of Mathematical, Engineering and Management Sciences

Vol. 4, No. 3, 708-728, 2019

https://dx.doi.org/10.33889/IJMEMS.2019.4.3-056

Figure 9 shows a variation of the velocity of the fluid against the slip coefficient $\phi$ by taking different values of permeability parameter for constant values of $\mathrm{Re}, \mathrm{M}$ and $\eta$. It has been obtained that with the increase of permeability parameter $K$, the velocity of fluid increases sharply and forms a constant velocity at $\phi=1$.

Figures (10) and (11) show that for constant values of parameters $\eta$ and $\phi$, the velocity of the fluid decreases sharply with an increase of Reynolds number and permeability parameter but increases with the increase of magnetic parameter.

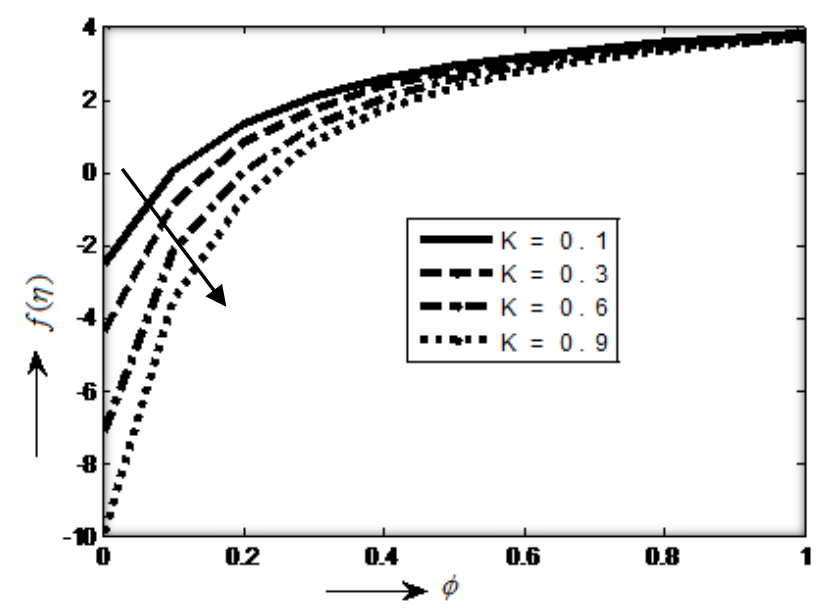

Figure 9. Variation of fluid velocity $f(\eta)$ against $\phi$ for permeability parameters $K(0.1,0.3,0.6,0.9)$ by taking parameters $\eta=1.6, M=2$ and $\mathrm{Re}=0.5$

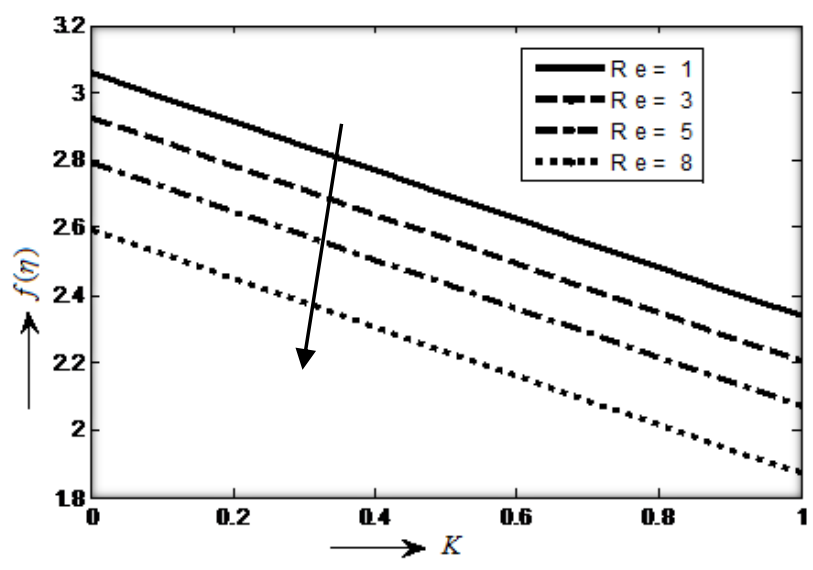

Figure 10. Variation of fluid velocity $f(\eta)$ against $K$ for Reynolds numbers $\operatorname{Re}(1,3,5,8)$ by taking parameters $\eta=1.6, M=2$ and $\phi=0.5$ 
International Journal of Mathematical, Engineering and Management Sciences

Vol. 4, No. 3, 708-728, 2019

https://dx.doi.org/10.33889/IJMEMS.2019.4.3-056

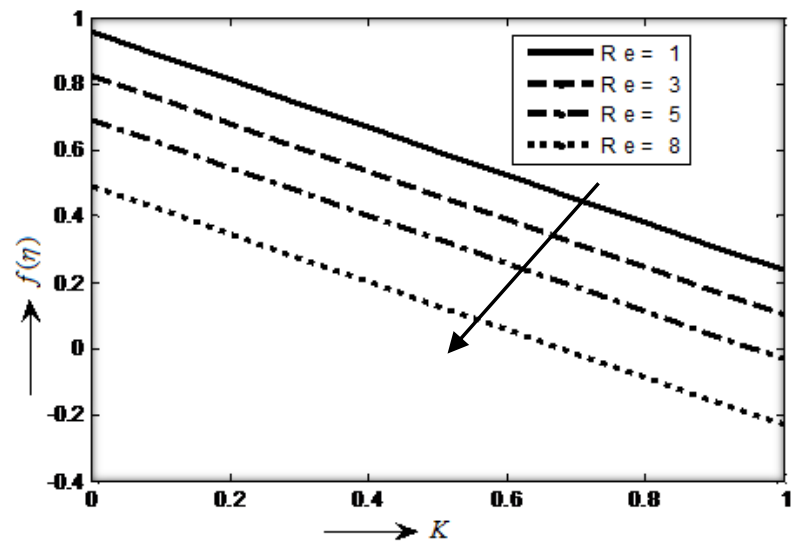

Figure 11. Variation of fluid velocity $f(\eta)$ against $K$ for Reynolds number $\operatorname{Re}(1,3,5,8)$ by taking $\eta=1.6$, $M=0$ and $\phi=0.5$

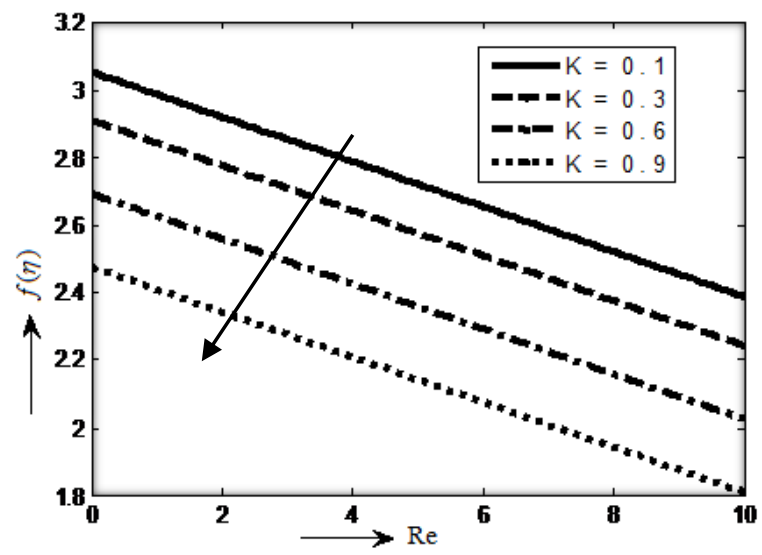

Figure 12. Variation of fluid velocity $f(\eta)$ against Re for permeability parameters $K(0.1,0.3,0.6,0.9)$ by taking parameters $\eta=1.6, M=2$ and $\phi=0.5$

The velocity of the fluid is plotted against Reynolds number for constant values of the parameters $\phi, M$ and $\eta$ in Figure 12 which shows that there is a sharp decrease in the velocity of the fluid in the channel as permeability parameter $K$ and Reynolds number Re increases.

The case $(M=0$ and $K=0)$ : In the absence of the magnetic field and the permeability parameter, the velocity of fluid has been plotted in figures 13 and 14 against the distance $\eta$ for different values of slip coefficient $\phi$ and Reynolds number Re. These graphs reveal that the velocity of the fluid increases with the increase of slip coefficient but it decreases as the distance from the nonporous wall increases. These graphs also show that the velocity of the fluid decreases with the increase of Reynolds number but it increases first and then decreases as the distance from the non-porous wall increases. Similar results for the velocity have been seen with the enhancement of Reynolds number Re for the constant value of slip coefficient which is shown in Figure 15. These results are similar to those obtained by Bujurke et al. (2010). In Figure 16, the velocity of 
International Journal of Mathematical, Engineering and Management Sciences

Vol. 4, No. 3, 708-728, 2019

https://dx.doi.org/10.33889/IJMEMS.2019.4.3-056

the fluid $f(\eta)$ is plotted against $\eta$ with $M=0, K=0$ and at $\phi=0.9$. It has been found that the velocity of the fluid decreases sharply with the increase of Reynolds Number Re which is opposite to that obtained in Figure 14 due to effects of slip coefficients because in Figure 16, the slip coefficient is $\phi=0.9$ whereas in Figure 15, the slip coefficient $\phi$ is less than 0.9. The overall observations of our paper match with the results described by Bujurke et al. (2010) in the absence of magnetic field.

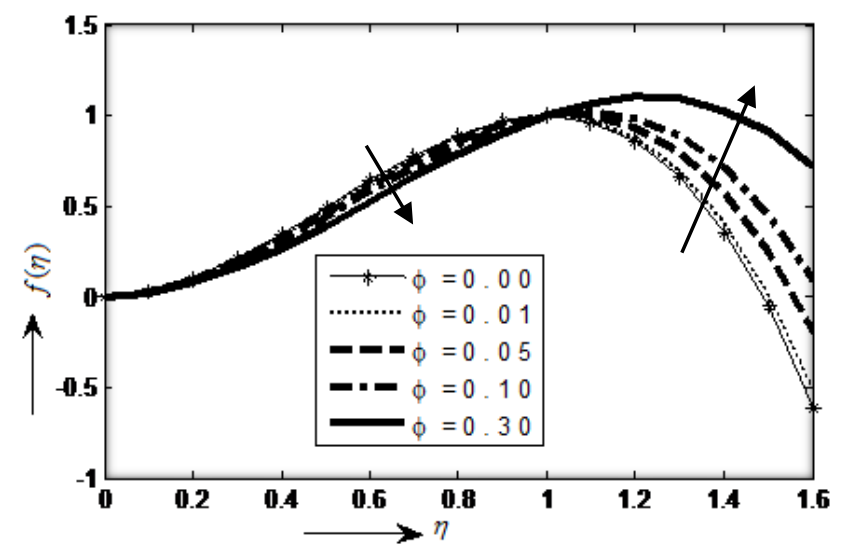

Figure 13. Variation of fluid velocity $f(\eta)$ against $\eta$ for slip coefficients $\phi(0.0,0.01,0.05,0.10,0.30)$ by taking parameters $M=0, K=0$ and $\operatorname{Re}=0.3$

Our problem describes the significant effects of permeability parameter and slip coefficient on the velocity of the viscous fluid flowing in a channel with a porous bounding wall in the presence of transverse magnetic field. This result also matches with the result of Naikoti and Vadithya (2014).

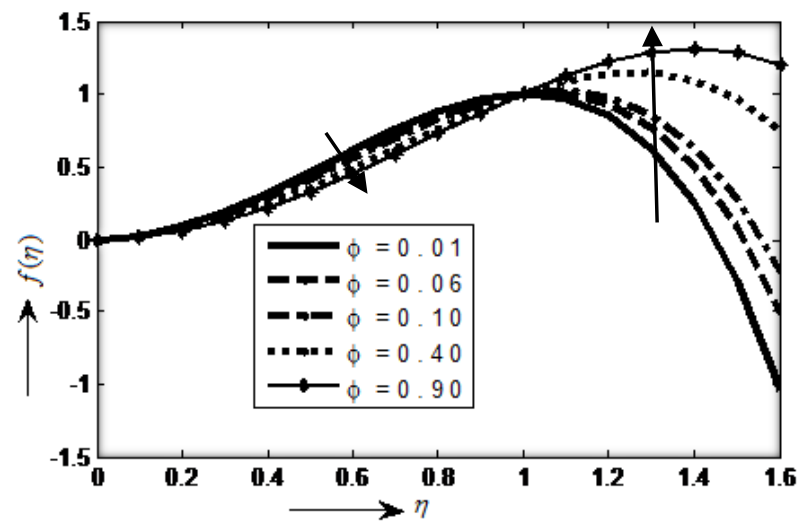

Figure 14. Variation of fluid velocity $f(\eta)$ against $\eta$ for slip coefficients $\phi(0.01,0.06,0.10,0.40,0.90)$ by taking parameters $M=0, K=0$ and $\operatorname{Re}=0.3$ 
International Journal of Mathematical, Engineering and Management Sciences

Vol. 4, No. 3, 708-728, 2019

https://dx.doi.org/10.33889/IJMEMS.2019.4.3-056

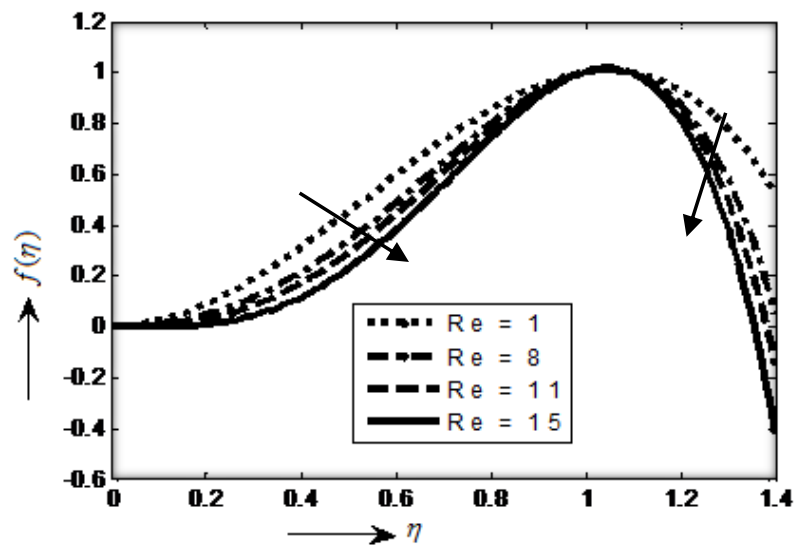

Figure 15. Variation of fluid velocity $f(\eta)$ against $\eta$ for Reynolds numbers $\operatorname{Re}(1,8,11,15)$ by taking parameters $M=0, K=0$ and $\phi=0.05$

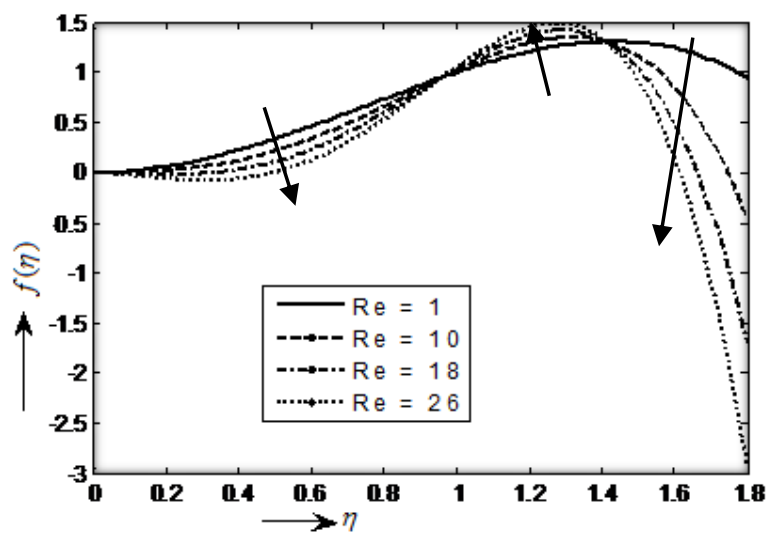

Figure 16. The graph of fluid velocity $f(\eta)$ against $\eta$ for Reynolds numbers $\operatorname{Re}(1,10,18,26)$ by taking $M=0, K=0$ and $\phi=0.9$

\section{Conclusion}

The effect of a transverse magnetic field on the laminar flow of viscous incompressible fluid in a channel with a porous bounding wall of different permeabilities has been analyzed. The analytical expression for the velocity of the fluid has been determined by solving the non-linear differential equations using Perturbation method. The approximations obtained by this method are valid not only for the small values of the parameters but also for very large values of the parameters thus providing an efficient method for exact and numerical solution of non-linear differential equations. It has been found that with the enhancement of magnetic parameter (Hartmann number) $M$, the velocity of fluid increases sharply whereas the velocity of the fluid decreases with the increase of permeability parameter $K$. Due to upmost importance of Reynolds number, the 
International Journal of Mathematical, Engineering and Management Sciences

Vol. 4, No. 3, 708-728, 2019

https://dx.doi.org/10.33889/IJMEMS.2019.4.3-056

observations with the different Reynolds number on the velocity of fluids under the constant magnetic field have been also seen. It is found that the Reynolds number sharply affects the velocity of the fluid. Due to the gradual increase of the slip coefficient $\phi$, the velocity of fluid increases.

\section{Conflict of Interest}

The authors declare that there is no conflict of interests regarding the publication of this paper.

\section{Acknowledgement}

The authors thank the reviewers for their valuable suggestions which led to definite improvement in the paper.

\section{References}

Abdou, M.M.M., El-Kabeir, S.M.M., \& Gorla, R.S.R. (2007). Effect of mixed thermal boundary conditions and magnetic field on free convection flow about a cone in micropolar fluids. International Journal of Fluid Mechanics Research, 34(5), 387-402.

Abou-Zeid, M.Y. (2009). Numerical treatment of heat and mass transfer of MHD flow of carreau fluid with diffusion and chemical reaction through a Non-Darcy porous medium. The Open Mathematics Journal, 2, 22-35.

Akeem B. Disu, Christie, Y. Ishola \& Toyin Olorunnishola (2014). Heat and mass transfer on MHD oscillatory slip flow in a channel filled with porous medium. IOSR Journal of Mathematics, 10(4), 6977.

Berman, A.S. (1953). Laminar flow in channels with porous walls. Journal of Applied physics, 24(9), 12321235 .

Bujurke, N., Katagi, N.N., \& Awati, V.B. (2010). Analysis of Laminar flow in a channel with one porous bounding wall. International Journal of Fluid Mechanics Research, 37(3), 267-281.

Dey, D. (2019). Mixed convective MHD micro-polar fluid flow in a porous medium with radiation absorption. International Journal of Mathematical, Engineering and Mathematical Sciences, 4(2), 387399.

Ganesha, S., \& Krishnambal, S. (2006). Magneto hydrodynamic flow of viscous fluid between two parallel porous plates. Journal of Applied Sciences, 6(11), 2420-2425.

Hosseini, M., Sheikholeslami, Z., \& Ganji, D.D. (2013). Non-Newtonian fluid flow in an axisymmetric channel with porous wall. Propulsion and Power Research, 2(4), 254-262.

Jafaryar, M., Farkhadnia, F., Mohammadian, E., Hosseini, M., \& Khazaee, A.M. (2014). Analytical investigation of laminar flow through expanding or contracting gaps with porous walls. Propulsion and Power Research, 3(4), 222-229.

Mahanta, P.K. (2012). Numerical study on heat transfer of Non-Newtonian fluid flow over stretching surface with variable viscosity in uniform magnetic Field. International Journal of Scientific \& Research Publications, 2(12), 1-9.

Makinde, O.D. \& Osalusi, E. (2006). MHD steady flow in a channel with slip at the permeable boundaries. Romanian Journal of Physics, 51(3-4), 319-328. 
International Journal of Mathematical, Engineering and Management Sciences

Vol. 4, No. 3, 708-728, 2019

https://dx.doi.org/10.33889/IJMEMS.2019.4.3-056

Muthuraj, R., \& Srinivas, S. (2009). Influence of magnetic field and wall slip conditions on steady flow between parallel flat wall and a long wavy wall with Soret effect. Journal of Naval Architecture and Marine Engineering, 6(2), 62-71.

Naikoti, K., \& Vadithya, M. (2014). Thermal radiation effects on magneto hydrodynamic flow and heat transfer in a channel with porous walls of different permeability. Thermal Science, 18(Supplement 2), S563-S572.

Prasad, B.G., \& Kumar, A. (2011). Flow of hydro magnetic fluid through porous media between permeable beds under exponentially decaying pressure gradient. Computational Methods in Science and Technology, 17(1-2), 63-74.

Ramadevi, B., Reddy, R.J.V., \& Sugunamma V. (2018). Influence of thermodiffusion on time dependent Casson fluid flow past a wavy surface. International Journal of Mathematical, Engineering and Mathematical Sciences, 3(4), 472-490.

Ramakrishnan, K., \& Sathish, E. (2018). Influence of porous parameter on MHD flow between porous walls of finite thickness. International Journal of Pure and Applied Mathematics, 119(13), 71-79.

Reddy, P.G., Reddy, T.S., \& Varma, S.V.K. (2017). MHD flow of a Newtonian fluid through a porous medium in planer channel. Global Journal of Pure and Applied Mathematics, 13(7), 3811-3832.

Terrill, R.M, \& Shrestha, G.M. (1964). Laminar flow through channels with porous walls and with an applied transverse magnetic field. Applied Scientific Research, 11(B), 134-144.

Terrill, R.M. (1965). Laminar flow in a uniformly porous channel with large injection. The Aeronautical Quarterly, 16(4), 323-332.

Terrill, R.M., \& Shrestha, G.M. (1966). Laminar flow through a channel with uniformly porous walls of different permeability. Applied Scientific Research, Section A, 15(1), 440-468.

Vafai, K., \& Kim, S. (1990). Fluid mechanics of the interface region between a porous medium and a fluid layer - an exact solution. International Journal of Heat and Fluid Flow, 11(3), 254-256.

Yadav, R., \& Joseph, V. (2017). Numerical investigation of heat transfer along symmetrical porous wedge and effects of radiation in the presence of variable fluid viscosity of flow. Applied Research Journal, 3(10), 295-303.

Zhang, Y., Lin, P., \& Si, X.H. (2014). Perturbation solutions for asymmetric laminar flow in porous channel with expanding and contracting walls. Applied Mathematics and Mechanics, 35(2), 203-220.

(c) (i) Original content of this work is copyright (C) International Journal of Mathematical, Engineering and Management Sciences. All rights reserved. Except of uses under a Creative Commons Attribution 4.0 International (CC BY 4.0) license at https://creativecommons.org/licenses/by/4.0/ 